\title{
New Understanding of the Draw of Xiangqi
}

\author{
Shi Wei-Jiang ${ }^{1 *}$
}

${ }^{1}$ Williams Law Office, New York City, NY, USA

*weijiangs@gmail.com

\begin{abstract}
This article explores the art of the draw of Xiangqi. In essence,Xiangqi draw is the symmetry break between the winning and losing, which makes the art of Xiangqi evolves and enhances the humanistic values of Xiangqi game. The deep charm of Xiangqi art lies in the mentality of the players, which would only be fully revealed in the round-robin system, because this would bring the Xiangqi game into a "n-body problem" and the complexity has increased greatly. But nowadays it becomes commenplace for organizers of high standard tournament of Xiangqi to adopt a knock-out system so as to simplify the competition to be the "two-body problem". The best Xiangqi draw percentage should be at the golden section of all games outcome of winning and losing, that virtue as Aristotle once proposed. In order to decrease higher draw percentage, a new initial arrangement of Xiangqi was proposed, thereby greatly increasing the complexity of Xiangqi game.
\end{abstract}

Keywords: Xiangqi, draw, games, artificial intelligence(AI), aesthetic practice, golden section, symmetry breaking, multi-body problem

\section{对象棋和棋的新认识}

\author{
施卫江 ${ }^{1 *}$
}

\author{
${ }^{1}$ 美国纽约市 威廉律师楼 \\ *weijiangs@gmail.com
}

\section{摘要}

该文探讨象棋和棋的艺术性，提出新的视点：和棋的本质是胜负局之间出现对称性破缺，从而使得象棋艺术进 化, 提升了象棋的人文价值蕴含。象棋艺术的深层鬼力在于对弯棋手们心态上的较量, 这唯有在循环赛制上才 能够充分现出来, 因为如此使象棋比赛进入了 “多体问题” 境界, 复杂性大增, 可惜现今一些高规格比赛常见 的是采用淘汰赛制, 与人机对抗一样, 仅停留在 “二体问题”上。和棋并非多多益善, 需要维持在恰当的比率, 最佳的和棋比率是在胜负局总数的黄金分割点上，这符合亚里士多德的 “美德即中道” 原理。最后为改革象棋 比赛出现和棋过多现象，提出了新的象棋初始布阵的方案，从而提高了象棋变化的复杂性。

关键词: 象棋，和棋，游戏，人工智能，审美实践，黄金分割，对称破缺，多体问题

\section{1. 人工智能时代象棋的价值}

人工智能 $(\mathrm{AI})$ 时代, 人类大大扩展了自己的智慧, 可以设法投入更多更好的 AI 设备, 取代了很多机械 刻板的人类劳作, 以填补 “被遗忘的中间地带”（埃 森哲），这在从前是难以想象的。

$\mathrm{AI}$, 简单来说, 就是运用计算机技术来模拟并实 现人脑思考过程的一种技术, 人工智能的效用还需要 经过人类智慧的实践来检验。人类智慧是生命存在的
最高心灵形式, 为了让人造的 “心灵” 尽可能企及真 实的最高心灵, 一个办法是, 将 AI 成果直接投入到 与人的对抗之中, 进行一番较量而检验。多年来人类 不解努力在棋类游戏领域以检验 AI 的效用： 1997 年 深蓝 AI 与国际象棋头号棋手卡斯帕罗夫大战而取胜; 2006 年浪潮天梭 AI 战平象棋头号棋手许银川; 2013 年 PONANZA 的 AI 战胜将棋名手佐藤慎一; 2016 年 3 月阿尔法狗击败围棋高手李世石；2017 年 5 月阿尔 法狗再次以 3:0 横扫围棋第一人柯洁; 2019 年 12 月 韩豆 AI 让子与李世石进行告别赛以 $2: 1$ 取胜; 今天 
象棋第一人王天一感叹, “在网上遇到强力的软件, 只能选择逃跑，根本没得下。”[1]

但就目前的进展看, 对抗和交流只限于人机对抗 的 “单元灵性”- - AI 一方不具有灵性, 尚未进入到 远为高级的多元灵性主体参与的模式之中。人机对抗 中 AI 之所以纷纷战胜人类棋手, 主要是依仗于其蛮 力计算力和储存力, 近年来大获发展的 AI 自我学习 程序又强化了这二种能力。

今天人工智能与人脑智慧进行较量, 是许多学科 专家关注的焦点。谈及人工智能发展与中国象棋的关 系, 纵横象棋棋坛达半个世纪的名将柳大华坦言: “人 工智能对象棋的发展弊大于利” [2]。在我看来, 弊 端主要可归结为象棋比赛上出现和棋太多!

与围棋相比, 象棋在棋盘上的复杂性果然远远不 及, 但是象棋 (包括国际象棋) 又有她的独特魅力, 这个魅力的外在形式就集中在围棋所没有的和棋结 局之上一一可以看作是, 胜负之间的对称性有了破缺 而出现和棋, 事物得以演化, 使之博弯复杂性延伸至 更为高级的战略决策上, 以至于策略溢出棋盘之外, 上升至高级精神性类型, 但须知: 围棋却没有复杂性 和合法性的 “盘外招” ! 遗憾的是, 对于象棋 (包括 国际象棋) 的和棋的重要性的理解和认识, 当今象棋 界、国际象棋界、人工智能界、博弯论界、哲学界尚 远远的不足一一人们普遍只谈论 AI 是如何的厉害, 但其前提只是简单地设定在单个的人机对抗上, 即仅 为 “二体问题”, 进而得出一个粗粘、浅薄的结论来: $\mathrm{AI}$ 远比人类强大、围棋远比象棋复杂! 然而几乎没有 人去思考: 怎么把象棋（包括国际象棋）的人机对抗 投入到由多位棋手参与的循环赛制之中? 即实践为 “多体问题” ? - - 这在 AI 界称为: 多智能体系统 (multi-agent system)。

由于人类对于自身智能的理解非常有限, 造就了 对于人工智能认识的有限。如何让 AI 去认识象棋循 环赛比赛过程中 (尤其是在赛程的后阶段) 某盘棋局 的和棋的价值? 譬如, 如何认识和棋得 1 分后每一方 棋手所能获取排位价值（通常还得比较小分）, 即和 棋 1 分对于如何影响棋手排位的权重系数? - - 这 太值得去探讨了! [3] 正是由于象棋 (包括国际象棋) 和棋所蕴含着丰富的人文价值却未能洞明, 以致于今 天一些高规格的象棋大赛上, 较普遍地采取淘汰赛制 来取代循环赛制, 从而使象棋、国际象棋类游戏尚未 深化到高级层次的多元缠绕关系的复杂性上, 审美实 践不够充分, 同时也使得 AI 对博弯的研究尚停留在 肤浅的层面上, 远未进入灵性较量之中。

可以说, 象棋的复杂性和魅力特别展现在其和棋 之上。和棋所占比率, 若少了则乏味 (胡荣华), 若 多了呢?

\section{2. 象棋和棋过多的沉闪}

围棋只有胜与负的结局, 下围棋就是孜孜矻矻去
争胜。而象棋则不同, 有了胜负和平局这二种决然不 同的结果，致使系统发生对称性破缺，系统得以进化 至人文意义的层面，游戏艺术得以升华至对局棋手的 精神特质状态, 从而大大增添了象棋的人文魅力。然 而成也萧何, 败也萧何, 随着人工智能的大力开发, 近年来象棋发展的趋势是, 高规格赛事上常常 “一团 和气”, 和棋多多, 如此使象棋的艺术魅力逊色了许 多, 因和棋过多, 则呈现 “破缺” 少之 “对称性” 来。

人们欣赏象棋棋艺, 希冀看到棋手们激情四射, 个性卓然的发挥, “在艺术中有性格的作品才是美的。” (罗丹) 如此精神特质促使事物朝着差异性方向去发 展, 差异性增大则促发对称性破缺, 即分出棋局胜负 来, 亦即系统发生分叉现象, 从而促使象棋艺术突现 而进化。但是和棋的首要本质是对称性, 下成和棋使 得对局双方没有分出高低来, 各得 1 积分, 相互间的 排名对比在较多情况下暂无大的变化, 这样的结局在 纯艺术角度上, 遏制了象棋发展的意境空间。

和棋过多现象也反映出象棋的弱项: 博峦树和状 态空间尚不够高大深厚。AI 专家说, 象棋的这二项数 值要比国际象棋来得强, 然而赛场上和棋率象棋比国 际象棋来得还要高。因为象棋中, 执行攻击任务的棋 子是车马炮兵, 它们总体兵力的攻击能力不算很强, 双方各自都有专司守卫职能的士和象, 有了他们的存 在, 就可以抵消掉一部分对手的进攻力量, 于是即使 己方的强子车马炮的力量及相互配合比对手稍微弱 些, 假如尽力与对手兑掉进攻子力, 防御还是可以抵 挡一番。

和棋过多的另一原因是, 尽管象棋的中国规 则对循环 “长打” 作了限制, 但是象棋局面上棋子布 阵的对称性和局面重现率 (一种变换对称性) 都要比 国际象棋来得大, 如此使象棋能够施展有效复杂度的 空间不大, 局面容易为弱手所掌控, 若犯错程度不高, 则容易导向和棋。

和棋过多, 还有部分原因是棋手消极求和、默契 和棋一一大多为了省事省心而懒惰, 往往出现在团体 赛事上居多——这好比棋手的公司 “持股” 份额少, 对待公司盈亏的责任心就小, 或许另一种可能是出于 某种不可告示的交易。

为了遏制象棋和棋过多的弊端, 曾有各种修改规 则的建议推出, 其中以 2006 年胡荣华的“和棋黑胜” 最为著称。经过实践, 其弊端也显露无疑, 取消了和 棋就削弱了象棋艺术之美, 笔者对此提出过尖锐的批 评意见 [4], 现今 “胡规” 只是通行在淘汰赛最后阶段 的超快棋决赛上。

\section{3. 淘汰赛制的缺陷}

为了减少消极和棋、默契棋、人情棋、非法幕后 交易, 近些年来, 无论在国内还是国外, 无论是象棋 还是国际象棋, 往往高规格个人赛事上较多地采纳淘 汰赛制，然而更多的新问题却由此产生了: 


\section{1. 艺术大美隐脢不彰}

因为比赛就成了简单的 “二体问题” , 纯粹是二 位棋手之间的较量, 没有了三体以上互为缠绕的复杂 性 (亨利・彭加莱论证了 “三体问题”没有确定的解, 即为高复杂性, 遑论三体以上的多体问题。), 于是 比赛成为简单的非合作的零和博亦, 就缺失人文价值 参与其中的交互主体性一一即如循环赛事上职业棋 手常说的 “比赛就是比心态”-—在精神意志层面上 斗智斗勇斗狡猾, 使得对弯者心灵充分交流而境界提 升。

\section{2. 人易为机器奴役}

既然淘汰赛的 “二体问题” 大大增加了确定性, 于是 AI 功能大显身手, 锋芒盖过人类, 凭借于 AI 棋 手就可以轻松地下 “背书棋” 。早期的博亦理论如策 梅洛定理就是框定二人棋局, 认定有确定性的解 [5], 之后, 冯 - 诺伊曼的 “极小极大值定理” 建立在概率 论上, 从而呈现 “非确定性” , 但是这概率是玩家能 够预测的, 所以此 “非确定性” 放在更高层次上又是 “确定性” 的, 如今 AI 技术大多基于此原理。然而 当象棋赛进入三名棋手以上的循环赛时候, 棋手间相 互缠绕问题使得棋手的心理因素释放出来, 而心理上 的各种因素通常是呈现非线性关系, 极难由 $\mathrm{AI}$ 来模 拟和效仿。例如, 伊曼努尔 - 拉斯克、胡荣华等极高 明的棋手经常会下出看似不高明的招法来, 而实际效 果却上佳, 这些可描述为: 欺招、拖刀计、引蛇出洞、 虚张声势等等。

\section{3. 艺术色调暗笑}

象棋的艺术魅力展示需要在多样性中才充分展 开, 为求多样性, 需要各种不同棋艺风格类型特长的 棋手尽可能多地进入各种排列组合、二二配对相遇上, 棋手之间可以充分交流、切磋、碰墥, 然而淘汰赛制 大大压缩了这样的机会。

\section{4. 心里压力甚大}

比赛中棋手的心里压力过大从而影响棋艺的正 常发挥, 因为淘汰赛仿佛就是在悬崖边上搏斗, 一失 手便掉入深渊, 再无将功补过、翻身获解放的机会。

\section{5. 比赛机会不均}

棋手参与比赛的机会很难被全部安排到均等对 待, 也就是缺乏公平性。在赛事前阶段不可避免要出 现若干 “死亡之组” , 常常由于高手与高手在初期相 碰, 使得部分高手早早遭淘汰, 而另有部分低手却独 享好机会分配在高手少的 “快活之组” ，幸运进入后 阶段赛程。

\section{6 . 赛程太长}

看似淘汰赛可以缩短赛程, 可是实际上登顶棋手 在整个征途中需要比常规循环赛制弯出多得多的棋 局, 才能最后胜出, 因为赛程到了后阶段, 必然遭遇 上不少水平相当接近的高手, 和棋的局数必定多多, 为了分出胜负就需多多加赛, 于是和棋出现的几率也 往往比循环赛上多得多。

\section{7. 冷门太多}

比赛的偶然性大增, 赛前预测往往会落空。冷门 迭爆, 黑马群起, 乱像纷纭, 头绪恫然, 棋迷观之犹 如走马灯似的眼花缭乱, 却难以找到“合理” 的感觉。

\section{4. 象棋和棋的最佳比率该多少?}

象棋的和棋少了不美, 多了亦不美, 那么该以多 少才恰如其分?

今天，中国棋界纷纷颂扬吴清源 “中的精神” , 慑于吴的威名, 人们盛赞其思想为 “完美和谐”、“不 阴不阳” , “阴阳思想的最高境界是阴和阳的中和” [6]。若按此, 象棋的和棋似乎就该多多益善, 或若有 改进者: 象棋的最佳和棋率该是半数! 果如此吗?

上述观点无非袭用儒家的 “中庸之道” , “中” 者 “不偏之谓中” ，其实质就是搞平均主义：均分天 下, 泯灭差别, 平平庸庸, 碌碌无为, 谨小慎微, 畏 首畏尾, 扼杀个性, 磨灭菱角一一看似为上等境界, 实乃庸人心境的投射！“庸” 者 “不易之谓庸”, “不 易” 即没有变化一一这样就背离了辩证法的精神: 运 动、变化、发展。《中庸》的一个核心内容是 “折中 致和”, 其实质是调和矛盾、和稀泥! 而不是辩证法 精神的扬弃。

唯唯诺诺者进言中庸美德, 然则, 举凡 “中庸” 者乃偏执均等, 动轩 “和合” 者即嗜好稳定, 高调 “统 一” 者则缺失分化, 强化 “安稳” 者而菒乏发展, 实 质沦为守旧、嫉妒、懒惰、庸俗! “折中致和” 思想 流传至象棋界精英头脑里, 典例即如许银川撰写的 《半半歌》: “半壁河山半攻守, 半争成败半悟道。” [7] 许银川对此理解为 “干什么事情都是一半比较好” - 一此句子是全称判断, 必然要上升为 “中庸之道”, 于是显得迂腐。反映在赛场上, 虽然许银川的棋艺被 公认为炉火纯青般的高超, 但是他在棋赛中下成的和 棋率也算是高的，可见，他的实力与成绩二者之间并 非 “和谐” 相称着。当然许银川只是职业棋手，人们 不必强求他享有高深的人文思想。

我们不妨运用系统科学理论来解剖中庸之道机 理: “中和” 即平衡、对称之谓也, “折中致和” 的 实质是致力于平衡对称, 亦即不寻求破缺, 无破缺即 无演化, 无演化即无进步, 即维持现状, 故持保守立 场。

创立 “审美游戏说” 的席勒和斯宾塞均认为, 人 
的艺术活动是一种以审美外观为对象的游戏冲动, 象 棋作为游戏的艺术也同样地让棋手们以审美外观的 冲动来竞技。在美学上事物若呈现同形重复的外观即 呈现为对称之美, 象棋比赛得到和棋的结局亦是具有 某种程度的美感形式, 因为对局的双方各得 1 分, 取 得了对称平衡。然而我们在下面将要分析到, 这 “中 和” 形式并非大美而只是小美：它构成的统一步调, 即为简洁之美。凡是对称性事物即为其在形式变换之 下保持若干质料不变换, 于是其美感味道就在于以较 少的质料使之获得轻快步调和捷径来通达目的的形 式, 但正因形式滞留在 “对称性” 味道上, 无 “破缺” 的韵味而呈现为稳定性和保守性, 即在形式上并无实 质性演化, “对称显然是一种多样性中的统一”, 然 而, “统一不可能是绝对的, 也不可能是一个形式; 一个形式是一个集合体, 它必须具有成份, 这些成份 的结合方式构成了形式的特性。” [8], 故 “中和” 以 一元形态的单调工稳封闭而显得枯燥乏味。

然而人的游戏决定了审美实践亟需自由意志投 入到艺术创作之中, 创作就是创新, 创新要达成对称 破缺的外观美感就须将审美实践主体处在兴发状态, 且是多元化形态。然而对于象棋游戏来讲, 主观上单 纯为着和棋的终极目的而求对弯, 博弯主体就处于静 怡之态的与世无争、安逸休闲, 遏止了通往精神活动 高级形式的创新和进取。

西方主流美学界认为, 自古典时代康德、席勒以 降, 认定游戏审美依赖于人的主观与客观、理性与感 性、形式与质料、精神与物质、理想与现实的共同存 在, 并且强调两者相互作用而结合起来, 这相结合并 非如中国众多学者所热衰的：须搞成 “中和” 样子, 以一元化为归宿; 相反是如席勒比喻的, 游戏为 “活 的形象” 所冲动, 这就特别需要审美兴发, 需要主体 自由意志的积极参与。在此可以表明, 审美实践就是 辩证法精神的二元性互动激荡, 是美感形式的进化的 程序。

赋予人文意义的二元化互动激荡还需要协调者 参与。在西方整个的人文价值大系统里, 公正 (正义) 这个德性范畴被捧为至高至圣的大秩, 早在古希腊时 代被各派智者、哲学家们广泛地探讨, 其中以亚里士 多德的论述最为出色。在亚氏看来, 公正被安置在各 条德性之上的 “协调者” 位置上, 从而提出了著名的 “美德即中道” 命题: 公正的中道是 “等比率均等” - 一中道法则的基本规定就是合乎比例的均等或几 何的均等, 绝非是安置在二个对立项参数的居中位置 上。亚氏论述道: “公正事物必定至少有四项。两个 是对某些人的公正, 两个是在某些事情中的公正。并 且对某些人和他们所有的事物两者间是相等的。” “因 为人和事之间的比值是相同的。 $A$ 对 $B$ 的比, 完全如 $C$ 对 $D$ 的比一样。再交替搭配, $A$ 对 $C$ 的比, 如 $B$ 对 D 的比一样。这也就是说整体对整体一样。这种搭配 影响着分配的结果。如若各项在这样的结合, 其结果 就是公正。”[9]
亚氏的公正由四个项来规定 “等比率均等”，凸 现了二元性互动的辩证法精神: 对人、对事分而论之, 主客相分离, 但又相互协调、牵制反馈, 如此来彰显 的恰是运动、变化、发展的绝对性。应用于象棋博弯 中, 游戏者欲获取纹枰对弯中在结局统计上具有优美 感形式, 就可以将待解的和棋与胜负之比的未知数放 入到亚氏的 “等比率均等” 公式之中, 去求解出 “中 道” 来。

于是我将亚氏的美德即中道 “等比率均等” 用数 学公式表达出来:

$$
A / B=C / D \text { 等价于: } A / C=B / D
$$

假设象棋比赛中: $\mathrm{A}$ 为和棋率, $\mathrm{B}$ 为胜负率, $\mathrm{D}$ 为 对局数整体率: 1 。由于待解的未知数仅为二元, 而象 棋比赛得分率的统计规则是, 某位棋手的和棋率加上 胜负率恒等于 1 , 因此 $A+B=D=1$ 。

由于亚氏所理解的 “对人与对事” 的二个项是联 动的, 是相互协调牵制的关系, 这让象棋比赛反映到 “等比率均等” 公式里, $\mathrm{B}$ 与 $\mathrm{D}$, 或 $\mathrm{C}$ 与 $\mathrm{D}$ 之间该是 怎样的关系呢?

辩证法乃持以永恒的动态来实践自我否定, 这是 运动、变化、发展的观点。阿多尔诺声称, 辩证法的 精神就是永恒的否定, 黑格尔说, 辩证法的扬弃就是 否定之否定。对于一个特定的审美价值系统, 以象棋 游戏为例, 美感要获取 “在场”, 需要系统进行持续 的对称性破缺, 对于美感存在形式现状要不断进行否 定。由于对局之前, 棋手双方的比分就是 “ $0: 0$ ”, 即 默认的 “平局” 状态, 对此现状既要进行 “否定” 而 扬弃, 也要进一步对待出现的胜负局进行再“否定”, 而这前后两个 “否定” 应该置于同等的重要性，也就 是说，对于胜负局进行辩证 “否定” 而达成和棋率， 与从对局整体局数中决出胜负局率，两者置于同等比 率，这样才算符合公正。

所以认定： $B=C$, 于是 “美德即中道” 可简化 为 “黄金分割率公式” $A / B=B / A+B$

由一元二次方程求根公式 $\mathrm{A}=\frac{-b \pm \sqrt{b^{2}-4 a c}}{2 a}$

知, $\mathrm{A}$ 有二个解, 通常用符号 $\Phi$ 来表示:

$\Phi_{1}=\frac{\sqrt{5}-1}{2} \approx 0.618$, 即和棋率对胜负率之比;

$\Phi_{2}=\frac{\sqrt{5}+1}{2} \approx 1.618$, 即胜负率对和棋率之比。

于是得出, 和棋数占整个对局数的最佳比率为:

$$
\Phi_{1} \approx 1-0.618 \approx 0.382 \text { 。 }
$$

为什么和棋率的最佳值应该约是 0.382 , 而不应 是 0.618 呢? 因为和棋的美感是对于胜负的对称破 
缺, 而胜负的美感就在于对于默认平局的破缺（尚未 对局之前就是双方默认的平局），其最优美值约为 0.618, 所以最佳和棋率也可以这样算:

$$
\Phi_{2} \approx 0.618 \times \Phi_{1} \approx 0.618^{2} \approx 0.382, \text { 与上述 }
$$
算法得出一致的结果。

可见，唯有黄金分割率才是 “天道至美”，这里 既是 “对称” 又有 “破缺” ，以二元性的动态开放姿 势来变幻而成 “气韵生动” , 乃将艺术创造处于兴发 状态, 是复杂性的 “在场” 体验和灵性建构。

人, 为了本真的生存, 乃 “诗意地栖居大地”, 在对待艺术创作上, 海德格尔论道: “作品本身越执 迷于它自己开启的敞开之境, 它就越单纯地让我们进 入此一敞开, 并因此而离开我们熟悉的意境。” [10] 审美实践活动即是通向 “澄明之境” 而可由优美感直 觉所引导, 为此需要获得 “轻松” 的愉悦感 (柏格森), 并为此而 “节省力气” 以摆脱繁琐 (斯宾塞) , 这些 都是为了 “无目的的合目的性” 的超功利性 (康德)。

人玩要游戏, 乃投入到美意诗情之中, 直觉体验 真实生命, 即 “存在得以呈现的场所”。对此, 海德 格尔论道: “真正的人应当是一个一切尚付阙如的 “此 在”, 审美活动就产生于这一 ‘此在”, 向真正的存 在敞开, 显现在澄明状态之中。在这里, “此在”不 是被决定、被限制的东西, 而是第一次成为自我决定 的东西。” 人们从事艺术活动, 正是为了更好地满足 自己对主观缺憾的慰籍需求并由此产生的情感上的 行为需求, 使得自己成全为一个“完整的人”(席勒)。

观照象棋艺术。象棋游戏可以看作为生命力盈余 的棋手用来体验 “此在” 、探寻自我所具有本真意义 的审美实践活动（席勒、斯宾塞）, 此时此刻, “此 在” 就是主观缺憾的慰籍需求而引发的寻受和满足的 欲念。从这个意义上讲, 最本质的存在就是 “自由”, 自由就是自我决定的动姿, 以自由的自我来不断地敞 开心扉, 走在通向 “澄明” 境界的心路上, 为此自我 希冀为生命价值的现状来一个升华, 反映在象棋游戏 的结局上, 就是对于默认 “平局” 现状感到缺憾, 期 盼着 “变化”, 希冀决出胜负来, 同时又不满足于停 留在胜负的现状, 持续深化 “对称性破缺” , 还希冀 和棋。

人玩要游戏, 是由审美实践在时间的“绵延之流” （柏格森）中去生化、再造、超越, 去体验生命的在 场性, 于是乎遵循生命在内的使命。生物学家证实, 许多生物生长的个体数之展开, 其规律恰是一个斐波 那契数列, 其内含有美妙无比的 “黄金分割率” , 此 即为自然选择的造化, 由此也似乎可与象棋游戏所内 涵着的胜、负、和的美德规律作一番类比。作为审美 实践活动的象棋游戏, 就是博弯主体不断扬弃、重塑 自我的努力, 乃自觉践行美学规律, 即以合乎 “美德 中道” 来行事。对于象棋和棋率最佳值的期盼, 亦可 看成是对黄金分割率所蕴含的天道至美进行 “内摹仿” （谷鲁斯）。

\section{5. 象棋改革的一种尝试}

象棋理论认为, 象棋之所以会出现胜负, 是由于 对弯双方中起码有一方行棋下出坏招, 即明显的失误 才破坏了局面的均势。因为象棋的初始布阵让双方完 全处于局面对称, 只是由于红先黑后行棋的次序, 才 导致局面在先后手上有微小的失衡。但是对亦进程到 残局后, 只要双方的强子尽可能多的兄掉, 而防守子 力士相尚健在, 仅由于先后手较小差距, 尚不致于导 致胜负的结局。

象棋特级大师一般有把握, 只要有充足的思考时 间就不容易出现行棋大失误，基本上可以找到较为精 确的应对着法, 若对弯双方均无大的失误便容易导致 和棋。有些高手甚至还掌握了 “经典和棋谱” , 只要 拿到红棋而思定和棋的话, 大致可以确保之。但若赛 场上和棋过多就会让人乏味, 于是人们不再热衰于象 棋游戏, 象棋艺术贬值。反映在系统论上, 和棋过多 就是呈现为对称多而破缺少, 这样系统的演化匮乏推 动力量就是难以升华至人文艺术佳境。

和棋过多逼迫象棋要改革, 近年来有不少倡议者 提出了多种方案, 譬如从国际象棋借来的种种: 相象 越过界河攻入对方阵地; 兵卒冲入底线立即升变; 马 腿不整住; 将帅出宫御驾亲征等等, 然而这些方案都 是难以让职业棋手去接受, 因为上述任何一种方案若 被采纳, 使象棋的行棋规则稍作更改, 这可谓牵一发 而动全身, 整套象棋的战略战术为之大大改观, 如此 对于一位职业老棋手而言，成年累月积聚的知识、训 练的心得、实战的经验、陶冶的谋略, 大多化为乌有、 灰飞烟灭, 况且新老战略战术交接还会引起思维混乱。

还有一种有效措施是, 减少双方比赛用时, 使得 棋手的临场发挥失误增多增大, 但却使得象棋的对亦 质量随之下降, 也算不上是好的办法。

另有倡议者提出 “大分小分调整法” 来计分: 当 和棋终局时候, 统计对弯双方各自余下的所有子力及 其相应的积分, 这里就有多种具体的方案, 其目的都 是用来搞清楚和棋时双方的优劣势对比, 从而打破均 势平衡的终局。显然易见，这些所谓的 “附加规则”

(此略) 极为繁琐且有很强的主观性成分（一个子力 的实际价值与其位置以及与己方其他子力的配合度、 控制对方的程度等等紧密相关），因而极不易操作。 更为欠缺的是, 由此降低了象棋的艺术性和优美感。 因为艺术性是与其质料的复杂性密切正相关, 而复杂 性又与非线性密切正相关, 非线性与非对称性密切正 相关, 然而当双方的终局计分最终还需要靠余下的子 力来评估作总计积分的时候, 就这项规则而言对称性 和线性度增加了, 亦即: 多子多分, 子越多分越高, 反之亦然。再说，艺术之美又与形式 “在场” 的简洁 性密切正相关, 而形式又与规则密切正相关, 因此, 规则繁琐则象棋艺术不美矣。

今天, 低等级分棋手只要拿到先进的 AI 软件就 无须消耗脑筋, 能够很快掌握开局至中局的各路变化, 
从而降低了象棋的复杂性。魔高一尺道高一丈, 象棋 改革的思路就是要增加其复杂性来抗衡, 对此特别需 要增加 “布局歧路”, 无冕之王贾题蹈在评注五羊杯 赛上柳大华对阵胡荣华的一局棋时这么论述：“应当 知道, 象棋最难处即在于歧路上掌握方向。所谓懂得 布局、善于布局, 就看有无针对双方的制约关系掌握 方向的能力。具有较高水平的棋手，临局犯错误大多 数是在通盘大意处迷失了方向, 对布局中的先后次序 出现了误差, 而技术上的漏失往往是偶然的。所以尽 管布局阶段就着法的度数来说不出十多个回合, 但由 于选择较自由, 歧路较多, 要能在所有岔路口上准确 地掌握方向, 严格来讲, 需要一眼能看几十着乃至百 步的工力才比较有把握。”[11]

同样的情况，国际象棋也面临着和棋过多的问题, 多年来有人提出要增加其复杂性的改革方案, 如前世 界棋王鲍比 - 菲舍尔提出过 “任意制国际象棋 (Chess960) ”, 前世界棋王克拉姆尼克提出 “取消 王车易位” 的着法, 等等, 但也是面临改革困境。

我的改革构思是从高丽象棋上获得灵感 (见: 图 1) ，设想一种新颖的 “象棋”, 它的初始布阵: 把 “帅”、“将” (与高丽象棋中对应的分别为 “楚”、 “汉”) 的位置放置在各自的九宫中央, 而不是现行 的九宫底线中心（见：图 2)，至于其他棋子的布阵 及行棋规则毫发未动, 故容易为职业棋手所接受。

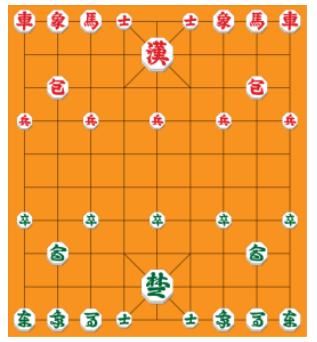

图 1 高丽象棋布阵

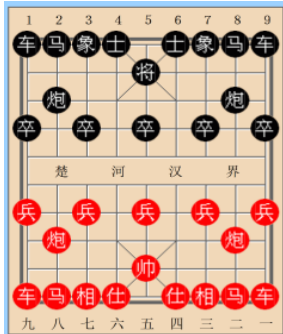

图 2, 新颖象棋布阵之一
在新布阵下，帅和将都被安置于风险之地，自然 增添了受攻击的危险性, 当然每一方都可以避险而把 将、帅缩回穴中, 但是需要付出一步棋的代价。这样 一来, 就得考验、同时也是增加给双方以勇气的机会, 也就是增加了 “布局歧路”。在双方勇气受鼓励的前 提下，增添了自由选择机会，增加了双方攻防之间态 势变换的复杂性, 于是决出胜负的概率提高。

从博弯树层面说, 新的布阵只是现行布阵的变体, 其他规则不变, 于是新布阵的博恋树覆盖了现行的, 可看成: 现行的博峦树是在新的博亦树上长出的枝干。 象棋的开局博亦树叶子节点呈现网络型结构, 次序颠 倒的走法往往可以异途同归, 因此新的博弯树可以与 现行的相嫁接, 其实是一个包含的关系, 博亦树和状 态空间都增大，于是复杂性增加。

现行的象棋执红棋的第一手棋, 职业棋手公认, 流行的合理走法有六种: 炮二平五, 炮二平四, 炮二 平六, 相三进五, 兵七进一, 马八进七。如今进入新 的象棋布阵, 就有了第七种走法: 帅五退一。“缩头”
使棋局复杂性增加而棋手犯错的几率增加, 于是和棋 有所减少。

进而, 新规则还可绑定红方 “帅” , 限定其在若 干个回合后才可松制行动, 以解决红黑棋之间先后手 差距的问题, 至于需要多少回合的松绑红帅, 使之达 成红黑双方之间在开局阶段的势力平衡? 这还有待 于实践检验来完善之。

另一种方案, 初始布阵仅单方让红方的帅放置于 九宫中心（见：图 3），不对行棋规则作其他的任何 改变, 就这项简单的改革, 可以使象棋的复杂性大为 增加，其改革的好处在于，遏制住红方的先手利益， 不过这将稍微破缺了双方布阵对称性的美感。

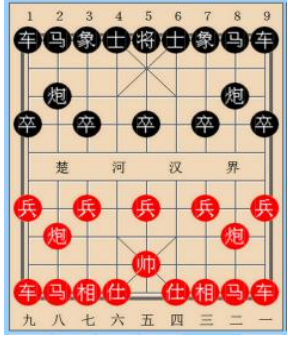

图 3, 新颖象棋布阵之二

\section{6. 结论}

作为系统对称破缺的形式，和棋应看成是象棋博 亦艺术进阶至人文高超境界的启动之源, 人文精神是 难以让 AI 技术来执行和表达的, 因为价值世界与事 实世界之间永远存在着鸿沟（休谟）。象棋游戏应发 挥出至高至深的人文精神来摆脱 AI 的霸权和僭越, 为此须安置象棋于多体的对抗较量之中, 也就是适用 于循环赛制, 回避 “二体博栾” 的淘汰赛制。这样, 棋手们就进入了列国纷争的 “多体博弯” 中，复杂性 陡增使得棋手纷纷去寻求各自的均衡点。

象棋艺术欲呈现大道至美的境界, 须设法调整游 戏规则, 让象棋的和棋率稳定在整个赛场棋局数的黄 金分割点附近。本文巧妙地运用数学方式来论证美学 上的一个著名命题。

为减少象棋和棋过多现象，本文设想一种改革方 案, 使游戏复杂性增加而可操作性又较强, 即参照高 丽象棋而建立起新的象棋初始布阵来。

\section{REFERENCES}

[1] Author (unknown), 2017. Players equipped with software, beg your pardon, don't insult Xiangqi on the live broadcast platform! http://sports.sina.com.cn/go/2017-08-18/docifykcypp9240732.shtml

[2] Wen Xuan, (2019) Liu Dahua: AI of Xiangqi does more harm than good and it ruins art appreciation of Xiangqi. http://sports.sina.com.cn/go/2019-0118/doc-ihqfskcn8351518.shtml 
[3] Shi Weijiang, (2018) On the complexity in decision making of Xiangqi. In: ICHSSR • 2018. Wuxi China

[4] Shi Weijiang, (2007) The new rules stifles the artistic charm of Xiangqi

http://www.gdchess.com/bbs/dv_rss.asp?s=xhtml... 52\&boardid $=40 \& \mathrm{id}=21503$ \&page $=47 \&$ star $=1$; http://blog.sina.com.cn/s/blog 4c079be20100090j. $\underline{\mathrm{html}}$

[5] Ulrich Schwalbe ${ }^{1, a}$, Paul Walker ${ }^{2, b},(1999)$. Zermelo and the Early History of Game Theory. http://abel.math.harvard.edu/ elkies/FS23j.03/zerm elo.pdf

[6] Wu Qingyuan, (2016) Autobiography, CITIC Press Corporation, Beijing. p.215.

[7] Shi Shaozong, (2009) Xu Yinchuan talks about his own thinking about Xiangqi: the way of Xiangqi is like the way of life http://news.hqcr.com/projects/news/view.aspx?nid= $\underline{93688}$

[8] George S, (1896) THE SENSE OF BEAUTY, www.iupui.edu/ $\sim$ santedit/sant/ P.63.

[9] Aristotle, (Second Edition 1999) Book V Chapter 3, Nicomachean Ethics, Hackett Publishing Company, Inc. Indianapolis/Cambridge. pp. 71-72.

[10] Heidegger M, (1971) Poetry Language Thought. Harper \& Row Publishers, Inc. New York, p.66.

[11] Jia Titao. (1998) The 26th round, Liu Dahua vs. Hu Ronghua, Refined Explanation of Famous Xiangqi Game. Shurong Bridge \& Chess Press, Chengdu. Scanning edition P.134. 